\title{
Robust Control of Ultrasonic Motor Operating under Severe Operating Conditions
}

\author{
Moussa Boukhnifer \\ Laboratoire Commande et Systèmes ESTACA, F-92300 Levallois-Perret \\ France \\ Antoine Ferreira and Didier Aubry \\ PRISME Institute ENSI Bourges, F-18000 Bourges \\ France
}

\begin{abstract}
Ultrasonic motor technology is a key system component in integrated mechatronics devices working on extreme operating conditions subjected to thermal cyclings or large thermal variations, EM disturbances, radiations, corrosion, or strong vibrations. Due to these constraints, robustness of the mechanics/electronics/control interfaces should be taken into account in the motor design. A robust controller for a travelling wave ultrasonic motor (TWUM) is considered in this study for operation in extreme environmental conditions. A simple causal model of the TWUM is introduced for identification of motor parameters. Then, an $H_{\infty}$ loop shaping synthesis procedure is implemented in order to obtain a good compromise between environmental robustness and motor's performances. Finally, simulation and experimental results demonstrate the effectiveness of the proposed robust controller in extreme operating conditions.
\end{abstract}

Keywords $-H_{\infty}$-synthesis, extreme environments, robust control, traveling wave ultrasonic motor.

\section{Nomenclature}

\begin{tabular}{|c|c|}
\hline \multicolumn{2}{|c|}{ SymbolDescription } \\
\hline$S_{i, j}, D_{n}, \Sigma$ & Strain, Electrical displacement, Entropy \\
\hline $\mathrm{T}_{i, j}, E_{n}, \theta$ & Stress, Electrical field, Temperature \\
\hline $\mathrm{s}_{i, j, k, l}^{\theta, i}$ & Elastic compliance \\
\hline$\epsilon_{n, m}^{T, \theta}, d_{n, i, j}^{\theta}$ & Electrical permittivity and piezoelectric constant \\
\hline $\mathrm{p}_{m}^{T}, \alpha_{i, j}^{E}$ & Pyroelectric and thermal expansion constant \\
\hline$\rho \frac{C^{T, E}}{\theta}$ & Thermal constant \\
\hline$(\alpha, \beta),(d, q)$ & Stator's and rotor's reference frames \\
\hline$\underline{\mathrm{v}}, \underline{\mathrm{w}}, \Psi(\underline{v}, \underline{w})$ & Voltage phasor, Rotating traveling wave, Phase \\
\hline$\theta_{c}, k, R\left(k \theta_{c}\right)$ & Angular position,Contact points,Rotational matrix \\
\hline $\mathrm{V}_{\text {Nid }}, V_{T i d}$ & Ideal normal and transversal velocity \\
\hline $\mathrm{V}_{d}, V_{q}$ & Voltage in the $(d, q)$ rotor's reference frame \\
\hline $\mathrm{F}_{N}, F_{T}$ & Normal and tangential force \\
\hline $\mathrm{N}, \mathrm{T}, \mathrm{f}_{0}$ & Real rotor speed, Torque, Friction coefficient \\
\hline $\mathrm{f}_{r \alpha}, f_{r \beta}$ & Feedback forces in the $(\alpha, \beta)$ 's reference frame \\
\hline $\mathrm{A}, \mathrm{m}$ & Force factor, Modal mass \\
\hline $\mathrm{d}_{\mathrm{s}, \mathrm{c}}$ & Stator's damping term and stiffness \\
\hline
\end{tabular}




\section{Introduction}

Nowadays, the market for mechatronics systems in such high tech sectors as aeronautics, aerospace, automotive or defense is booming. In those expanding industrial sectors, those mechatronics systems are put to the test of extreme operating conditions such as thermal cyclings or large thermal variations, radiations, corrosion (5), demanding vibratory environments for their mechanics and their electronics, intense pressures or ultra high vacuum conditions (1), sharp accelerations, or even more severe, high shocks. As a result the resistance and the robustness of those embedded mechatronics systems prove to be of paramount importance with respect to their harsh operating environments. In such a context, the ultrasonic motor technology suits mechatronics purposes perfectly due to its powerful performances to compare with its electrical servomotor counterparts. Indeed, its main characteristics are compactness, high torque at low speed without gears, high holding torque without power, low power consumption, fast response. Thus, the ultrasonic motor (USM) has already achieved to find its place in such industrial sectors. In particular, the traveling wave ultrasonic servomotor (TWUM) has already been tested recently in aerospace by the NASA to operate in cryogenic temperature (17) as well as in ultra high vacuum conditions (1). Moreover for aeronautics, in the current " fly-by-wire " era, some authors developed ultrasonic motor control strategies so as to implement active control sticks which allow the pilot to feel the force feedback from the rudders (2),(7).

Numerous TWUM control strategies have already been implemented in the literature. The speed control strategy (3) with one of the TWUM natural variables (frequency, voltage or phase difference) is the most classical one. However, it is very sensitive to the temperature variations and motor's performances are not optimized. Another strategy consists in controlling the wave amplitude thanks to the integrated piezoelectric sensor. Though this control is more thermally stable, it is still sensitive to torque variations. At last, authors have developed a TWUM model leading to the stationary waves control (7)(8)(9). This control resolves the previous control issues through frequency adaptation, wave phase and amplitude control. Unfortunately, it is a very complicated strategy to implement. It appears in view of this brief introduction that the TWUM servomotor's control is not straightforward, even more, when submitted to harsh environmental conditions. Thus, in section II the ins and outs of the TWUM behaviour will be exposed. The ultrasonic motor model selected will be presented briefly in section III. Section IV will present a robust control strategy simulation implemented in order to take account of the parameters and model uncertainties, load disturbances such as sharp torque variations and noises rejections. Finally, we will conclude on the adequacy of the $H_{\infty}$ robust control for the TWUM ultrasonic motor type in the context of an harsh operating environment.

\section{Ultrasonic Motor Sensitivity}

The travelling wave ultrasonic motor operating principle is based on the combination of two modes of energy conversion. Firstly, an electromechanical one, where a piezoelectric ceramic is excited at its mechanical resonance through an high frequency electrical supply. Secondly, the stator's minute elliptical motions resulting from the previous conversion are converted into rotation or translation through friction-driven transmission at the stator/rotor interface. So, as it will be further explained in the following paragraphs, due to its quite complex operating principle the TWUM control presents a real challenge within extremes and hostiles environments. Indeed, its behavior changes considerably when subjected to different exogenic 
energy sources. Furthermore, intrinsic nonlinear phenomenon, hysteresis and frequency shift occur in both energy conversion processes.

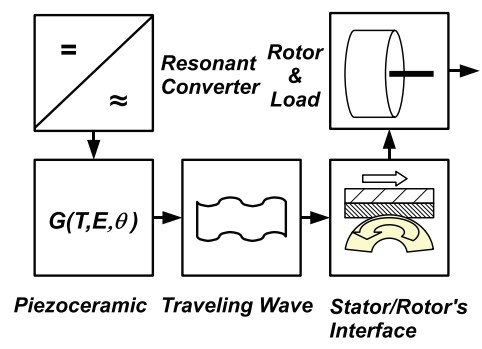

Fig. 1. Block diagram of the TWUM mechatronic system.

The electromechanical energy conversion, presents large parametric variations at the ceramic level, related to the origin of the piezoelectric phenomenon. Actually, when subjected to parasitic energy such as voltage surge, high mechanical stress, high loads or overheating, the piezoelectric ceramic naturally tends to minimize its potential energy $G(T, E, \theta)$ through local polarisation reorientation, hence it results a global parametric variation. For instance, the pyroelectric coefficient $p^{T}$ or the thermal expansion one $\alpha^{E}$ (Cf. Equations (1a),(1b),(1c)) vary greatly according to the temperature or under the motor own friction losses. Henceforth, the ultrasonic motor driving point shifts causing the resonance frequency which is traditionally a control input to drift. Thus, it appears that thermal variations have a great influence on the ultrasonic motor speed. Moreover, the presence of integrated vibration piezoelectric sensors, makes the ultrasonic motor control very sensitive to noises and disturbances. Lastly, the nonlinear and the hysteresis behavior of some of its parameters, as for example the electric permittivity $\epsilon^{T, \theta}$ or the piezoelectric constant $d^{\theta}$ (Cf. Equations (1a),(1b)), has an important and direct influence on the travelling wave ultrasonic motor control. Indeed, it appears that the resonance peak is not perfectly symmetrical. But, there exist in the lower frequency range a steep drop of the vibration velocity called the pull-out phenomenon. Therefore, owing to the frequency drift, we must absolutely take into account this phenomenon because it results in motor sharp speed drop.

$$
\begin{array}{r}
d S_{i, j}=s_{i, j, k, l}^{\theta, E} d T_{i, j}+d_{n, i, j}^{\theta} d E_{n}+\alpha_{i, j}^{E} d \theta \\
d D_{n}=d_{n, i, j}^{\theta} d T_{i, j}+\epsilon_{n, m}^{T, \theta} d E_{k}+p_{n}^{T} d \theta \\
d \Sigma=\alpha_{i, j}^{E} d T_{i, j}+p_{n}^{T} d E_{n}+\rho \cdot \frac{C^{T, E}}{\theta} d \theta \\
i, j, n, k=1 \text { to } 3
\end{array}
$$

Piezoelectric Equations

The second power conversion purpose is to convert the travelling wave wobbles induced by the piezoelectric ceramic, in rotational motion. The travelling wave wobbles result from the 
ceramic excitation at its mechanical resonance through a two-phase power supply switching within the ultrasonic range (between $40 \mathrm{kHz}$ and $45 \mathrm{kHz}$ ). In the piezoelectric motor case the power transmission is not so simple. Indeed the mechanical power transmission, for which the rotor is constricted on the stator thanks to a spring, comes from the mechanical contact at the stator/rotor interface. Classically, in order to enhance the best contact transmission and therefore the highest torque, an elastic layer is inserted on the rotor surface. Unfortunately, owing to complex forces interactions at the stator/elastic layer/rotor interfaces, it appears extreme nonlinear contact mechanisms between them (7). It should be notice that those nonlinear mechanisms are magnified over load variations or torque perturbations. Furthermore, due to feedback from contact forces, the travelling wave form is no more sinusoidal as in the ideal case. Equally, within the pull-out phenomenon drop zone, it may appear a hysteresis effect depending on the frequency directional variation (9). Finally, it results from this frictional power transfer a thermal dissipation which alters the piezoelectric ceramic parameters.

\section{Ultrasonic Motor Modeling}

\subsection{General Layout}

With the objective to implement a robust control strategy for the travelling wave ultrasonic motor, the motor model considered must be neither too much simplistic so as to take accurately account of its nonlinear characteristics, nor overly realistic and consequently hard to implement ; for instance, on a Digital Signal Processor (12) or on a Field Programmable Gate Array (11). Usually, the ultrasonic motor speed control is implemented and achieved thanks to the equivalent electromechanical model. However, the usual equivalent electrical model though generally sufficient to model the steady-state operation does not allow to accurately model the transitory operation. Furthermore, that speed control strategy is very sensitive to thermal and torque variations. Henceforth, some authors developed recently an original causal TWUM model which meets this study objectives. Indeed, this original model enables to set up torque control law with a relatively low complexity (2). Therefore, before tackling its robust control, the causal TWUM model will be presented afterwards as well as its assumptions and its advantages.

\subsection{Causal TWUM model in the stator's reference frame}

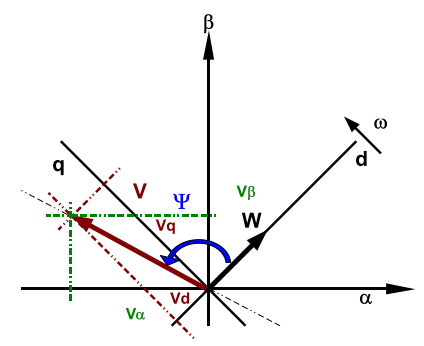

(a) stator's $(\alpha, \beta)$ and rotor's $(d, q)$ reference frame.

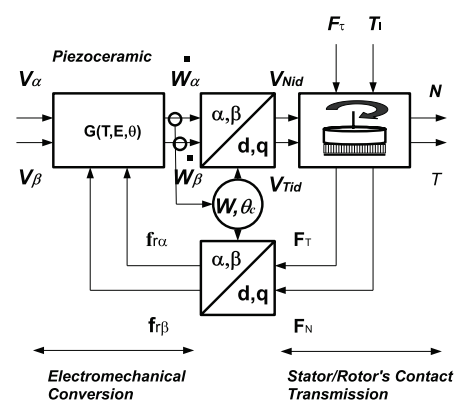

(b) Causal TWUM model.

Fig. 2. Causal TWUM model in the stator's $(\alpha, \beta)$ reference frame. 
The travelling wave ultrasonic motor is on its operating principle equivalent to an induction motor. Indeed, its principle is perfectly similar to the induction motor where the fluctuating magnetic field produced in the air-gap by the two-phase stator supply spawns rotor torque through induction. Consequently, some authors have applied the same mathematical formalism to express the TWUM model through space vectors, firstly in the stator's $(\alpha, \beta)$ reference frame, then in the rotating $(d, q)$ frame (as shown in Figure (2(b))). In a first stage, the twophase electrical supply, providing respectively the sinusoidal voltages $v_{\alpha}$ and $v_{\beta}$, feeds the two alternate piezoelectric sectors. It results from this supply two purely sinusoidal stationary waves, respectively $w_{\alpha}$ and $w_{\beta}$, expressed within the stator's $(\alpha, \beta)$ reference frame. The combination of those vibrating stationary waves propagates consequently along the stator, a rotating travelling wave $\underline{\mathrm{w}}$ forming in the $(\alpha, \beta)$ frame an angle $\Psi$ from the voltages phasor $\underline{\mathrm{v}}$ (Cf. Figure 2(a)). It is interesting to point out that the TWUM structure provides $k$ permanent contact points with the rotor, corresponding to the $k^{\text {th }}$ excited mode. Finally, the angular position $\theta_{c}$ and the wave crest $\widehat{w}$ are deduced in the $(\alpha, \beta)$ frame as follows:

$$
\begin{aligned}
& \tan \left(k \theta_{\mathcal{C}}\right)=\frac{w_{\beta}}{w_{\alpha}} \\
& \widehat{w}=\sqrt{w_{\alpha}^{2}+w_{\beta}^{2}}
\end{aligned}
$$

In a second stage, the travelling wave is in contact with a virtual rotor considered ideal. That's to say, that the $k$ contacts are considered punctuals with no slidings and no energy storing. This assumption at this point enhances, by means of the rotational matrix $R\left(k \theta_{c}\right)$, to express within the $(d, q)$ rotating frame the ideal transversal velocity $V_{T i d}$ along the quadratic axis $q$ and the ideal normal velocity $V_{N i d}$ along the direct axis $d$. Unfortunately, due to the elastic layer required to improve the contact transmission, the $k$ contacts are not ideal. Actually, the area at the stator/rotor is distributed and a sliding effect occurs, which is essential to provide torque similarly to the induction motor. Numerous authors have set about modeling this highly nonlinear contact transmission, resulting in sophisticated models with excellent accuracy. Still, in order to implement a straightforward model, despite the fact that the friction coefficient $f_{0}$ varies due to the nonlinear contact transmission, the relation between the real rotor speed $N$ and the torque $T$ is approximated and considered in the overall model as linear

$$
T=f_{0}\left(\frac{1}{b} V_{\text {Tid }}-N\right) \quad|T|<T_{\text {max }}
$$

In addition to the friction phenomenon, the TWUM requires so that to produce the torque $T$ and consequently to drive the load, a normal force $F_{N}$ to maintain contact condition at the rotor/stator interface as well as a tangential force $F_{T}$. The application of those mechanical stresses results in some feedback forces on the stator. Those feedback forces, respectively $f_{r \alpha}$ and $f_{r \beta}$, are then rotated from the rotating $(d, q)$ frame to the stator's $(\alpha, \beta)$ frame thanks to the previously used rotational matrix $R\left(k \theta_{c}\right)$. At this point, it seems important to notice that those feedback forces have a direct influence on the TWUM. Indeed, those external mechanical stresses provoke on the stator side, the piezoelectric ceramic energy to evolve and as a result the resonance pulsation $\omega_{r}$ to drift ; which is expressed in the causal TWUM model by $k \theta_{c}$ variations.

\subsection{Model's equations in the rotating $(d, q)$ reference frame}

The causal TWUM model expressed in the stator $(\alpha, \beta)$ reference frame enable to implement a straightforward control by meams of simplistic mechanical contacts. Nevertheless, it appears 
that the variable from the normal $\alpha$ axis and the tangential $\beta$ axis are coupled, which is shown through the rotational matrix $R\left(k \theta_{c}\right)$. Thus, so as to remedy to this variables coupling, the control is then given in the rotating reference frame. The feeding voltages $v_{\alpha}$ and $v_{\beta}$ are then deduced from $V_{d}$ and $V_{q}$ by means of the rotational matrix $R\left(k \theta_{c}\right)$ and the ultrasonic motor equations are deduced for small pulsation variations.

$$
\begin{gathered}
m V_{\text {Nid }}^{\cdot}+d_{s} V_{N i d}+\left(c-m\left(k \dot{\theta_{c}}\right)^{2}\right) \int V_{N i d} d t=A V_{d}-F_{N} \\
2 m V_{\text {Tid }}^{\cdot}+d_{s} V_{T i d}=A V_{q}-F_{T}
\end{gathered}
$$

In view of those equations it is interesting to notice that, due to reference frame change, the variables are no more coupled. Finally, those model's equations allow to determine the TWUM behaviour in the steady-state but equally the transitory. Thus, this straightforward model will be used in the next section in order to implement the TWUM robust control.

\section{Robust Control}

\section{1 $H_{\infty}$ Standard Problem}

For given $P(s)$ and $G>0$, the $H_{\infty}$ standard problem is to find the controller $\mathrm{K}(\mathrm{s})$ which :

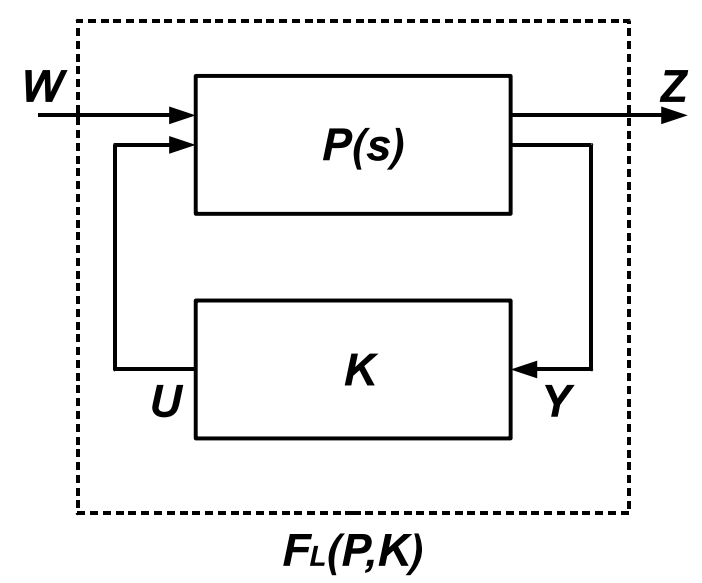

Fig. 3. $H_{\infty}$ standard problem.

- Stabilize the closed loop system in Figure 3

- Maintain the norm $\left\|F_{L}(P, K)\right\|_{\infty}<\gamma$

where $F_{L}(P, K)$ is defined as the transfer function of the outputs $Z$ according to the inputs $W$.

\section{2 $H_{\infty}$ Coprime Factorization Approach}

An approach was developed by Mc.Farlane and Glover (13)(14) starting from the concept of the coprime factorization of transfer matrix. This approach presents interesting properties and its implementation calls upon traditional control notions. 


\subsection{Robust Controller Design using Normalized Coprime Factor}

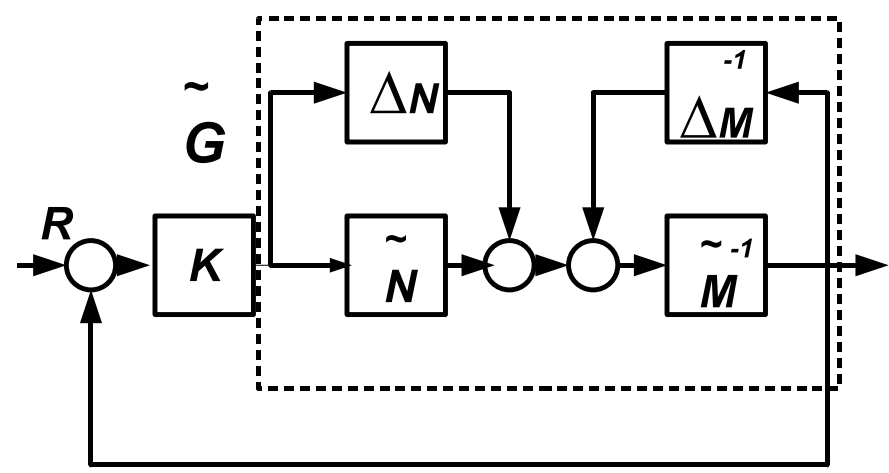

Fig. 4. Coprime factor robust stablization problem.

We define the nominal model of the system to be controlled from the coprime factors on the left: $G=\widetilde{M^{-1}} \widetilde{N}$. Then a perturbed model is written (see Figure (4)):

$$
\widetilde{G}=\left(\widetilde{M}+\Delta_{M}\right)^{-1}\left(\widetilde{N}+\Delta_{N}\right)
$$

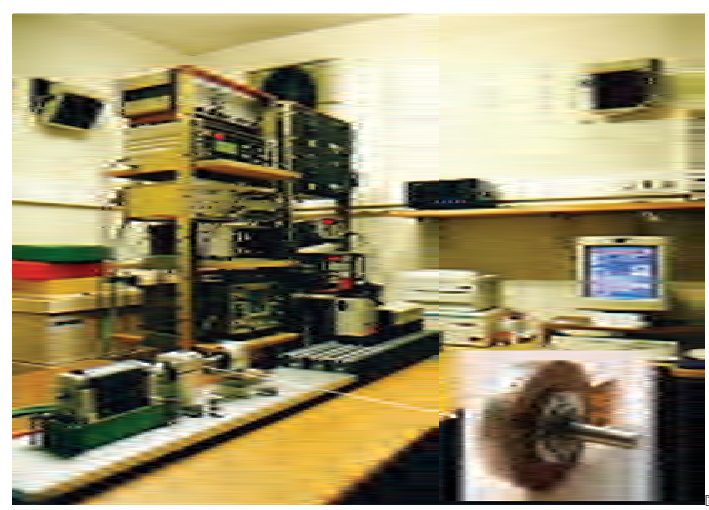

Fig. 5. Step response of motor torque.

where $\widetilde{G}$ is a left coprime factorization (LCF) of $G$, and $\Delta_{M}, \Delta_{N}$ are unknown and stable transfer functions representing the uncertainty. We can then define a family of models as follows

$$
\xi_{\epsilon}=\left\{\widetilde{G}=\left(\widetilde{M}+\Delta_{M}\right)^{-1}\left(\widetilde{N}+\Delta_{N}\right):\left\|\left(\Delta_{M} \Delta_{N}\right)\right\|_{\infty}<\epsilon_{\text {max }}\right\}
$$

where $\epsilon$ represents the margin of maximum stability. The robust stability problem is thus to find the greatest value of $\epsilon=\epsilon_{\max }$, so that all the models belonging to $\xi_{\epsilon}$ can be stabilized by the same corrector $K$. The problem of robust stability $H_{\infty}$ amounts finding and $K(s)$ stabilizing $G(s)$ so that :

$$
\left\|\left(\begin{array}{c}
I \\
K
\end{array}\right) \cdot(I-K \cdot G)^{-1}(I G)\right\|=\gamma_{\min }=\frac{1}{\epsilon_{\max }}
$$


However, Mc Farlane and Glover (17) showed that the minimal value of $\gamma$ is given by :

$$
\gamma_{\min }=\epsilon_{\max }^{-1}=\sqrt{1+\lambda_{\text {sup }}(X Y)}
$$

where $\lambda_{\text {sup }}$ indicates the greatest eigenvalue of $\mathrm{XY}$, moreover for any $\epsilon<\epsilon_{\text {max }}$ a corrector stabilizing all the models belonging to $\xi_{\epsilon}$ is given by :

$$
\begin{array}{r}
K(s)=B^{T} X\left(s I-A+B B^{T} X-\gamma^{2} Z Y C^{T} C\right)^{-1} \gamma^{2} Z Y C^{T} Z \\
=\left(I+Y X-\gamma^{2} I\right)^{-1}
\end{array}
$$

where $A, B$ and $C$ are state matrices of the system defined by the function $G$, and $X, Y$ are the positive definite matrices and the solution of the Ricatti equation :

$$
\begin{aligned}
A^{T} X+X A-X B^{T} B X+C^{T} C & =0 \\
A Y+Y A^{T}-Y C^{T} C Y+B B^{T} & =0
\end{aligned}
$$

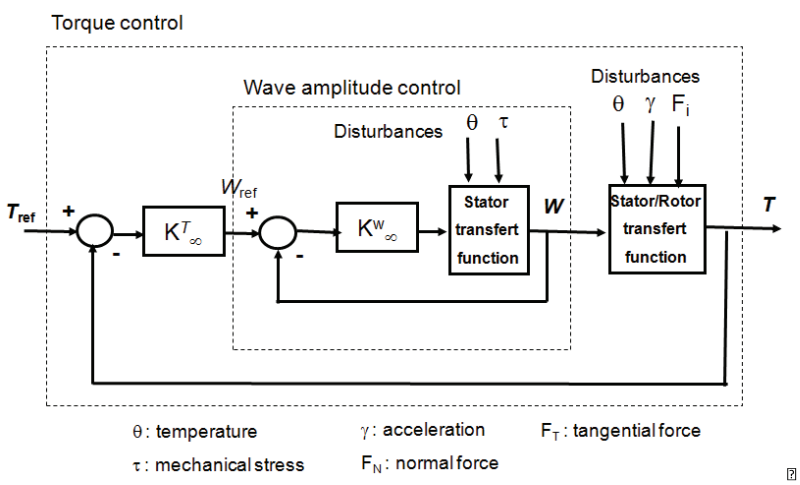

Fig. 6. Cascaded $H_{\infty}$ loop shaping controllers with inner-loop control $\left(H_{w}(s)\right.$ of the wave amplitude control of the stator) and outer-loop control (torque control loop).

\subsection{The Loop-Shaping Design Procedure}

Contrary to the approach of Glover-Doyle, no weight function can be introduced into the problem. The adjustment of the performances is obtained by affecting an open modelling (loop-shaping) process before calculating the corrector. The design procedure is as follows :
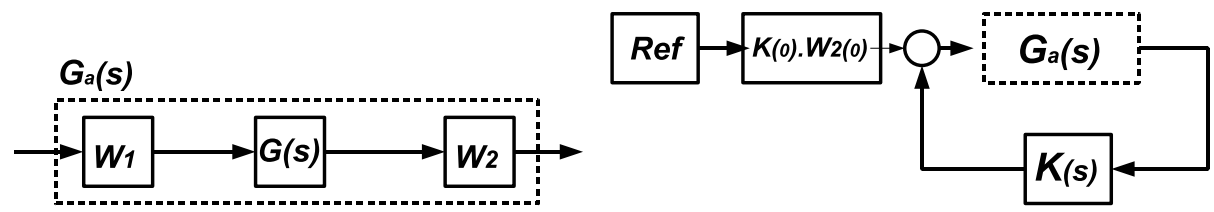

(a)

Fig. 7. The loop-shaping design procedure. 
1. We add to the matrix $G(s)$ of the system to be controlled a pre-compensator $W_{1}$ and/or a post-compensator $W_{2}$, the singular values of the nominal plant are shaped to give a desired open-loop shape. The nominal plant $G(s)$ and shaping functions $W_{1}$ and $W_{2}$ are combined in order to improve the performances of the system so that $G_{a}=W_{1} G W_{2}$ (see Figure $(7(\mathrm{a})))$. In the monovariable case, this step is carried out by controlling the gain and the phase of $G_{a}(j \omega)$ the Bode diagram .

2. From coprime factorizations of $G_{a}(j \omega)$, we apply the previous results to calculate $\epsilon_{\max }$, and then synthesize a stabilizing controller $K$ ensuring a value of $\epsilon$ slightly lower than $\epsilon_{\text {max }}$ :

$$
\|(K)\left(I-K W_{2} G W_{1}\right)^{-1}\left(I W_{2} G W_{1} \|_{\infty}=\gamma=\frac{1}{\epsilon}\right.
$$

3. The final feedback controller is obtained by combining the $H_{\infty}$ controller $K$ with the shaping functions $W_{1}$ and $W_{2}$ so that $G_{a}(s)=W_{1} G W_{2}$.(See Figure (7(b)))

\section{5. $H_{\infty}$ Loop Shaping Controller Design}

In this section, we present two cascaded $H_{\infty}$ loop shaping controller architecture composed of both inner-loop and outer-loop controllers. The inner-loop's one regulates in $H_{w}(s)$ the vibrational travelling wave amplitude $\hat{W}$ provided by the stator. The outer-loop controller as for it, ensures the torque feedback control of the motor shaft when subjected to variational loads.

\subsection{Wave Amplitude Control}

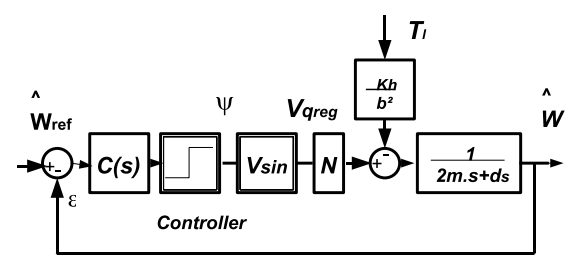

Fig. 8. Block diagram $H_{w}(s)$ of the wave amplitude control of the stator.

The first block of the control scheme of the Figure (8) regulates the wave amplitude. The transfer functions in the open-loop of the wave amplitude is given by (2):

$$
\widehat{W}=\frac{1}{w} \cdot \frac{N V_{q}-k \frac{h}{b^{2}} T_{i}}{\left(2 m+\left(k \frac{h}{b^{2}}\right)^{2} J\right) \cdot s+d_{s}}
$$

The synthesis of the wave amplitude controller $C(s)$ is obtained according to the implementation shown in the Figure (7) using the command ncfsyn of MATLAB $\mu$-Analysis and Synthesis toolbox (15). The controller $C(s)$ is obtained by combining the pre-filter $W_{1 m}$ and the post-filter $W_{2 m}$. The pre-filter and post-filter are used to shape the open-loop plant to achieve a desired frequency responses according to some well defined design specifications such as bandwidth and steady-state error (16). In order to ensure a high gain in low frequencies and a low gain 
in high frequencies and to obtain a high performance and a good robustness, we add the following weight functions.

$$
W_{1 m}=150 \cdot \frac{2 . s+200}{0.02 s+0.001} \quad W_{2 m}=1
$$

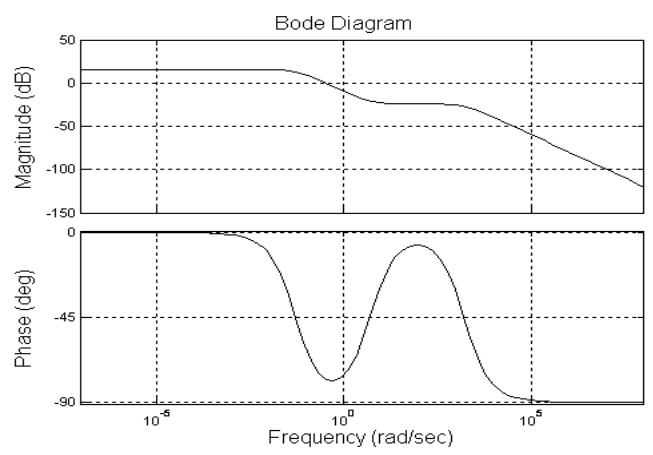

(a) Open-loop responses of $W_{m 1} P_{m} W_{m 2}$

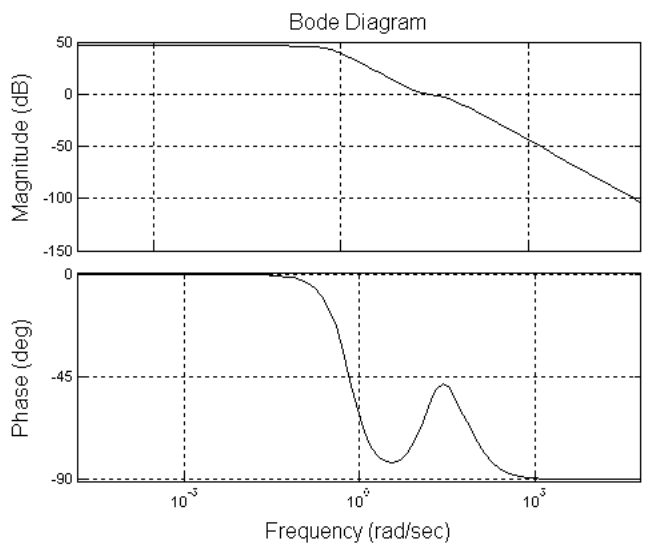

(b) Open-loop responses of $W_{m 1} P_{m} W_{m 2} C$

Fig. 9. Open-loop responses.

The Figures (9(a)-9(b)) shows the frequency responses of the system, the $W_{m 1} \cdot P_{m} \cdot W_{m 2}$ and the open-loop system $W_{m 1} \cdot P_{m} \cdot W_{m 2} \cdot C(s)$. The results show that the open-loop remains close to the step response obtained after the choice of the shaping functions and $C(s)$ ensures correct margins of stability 


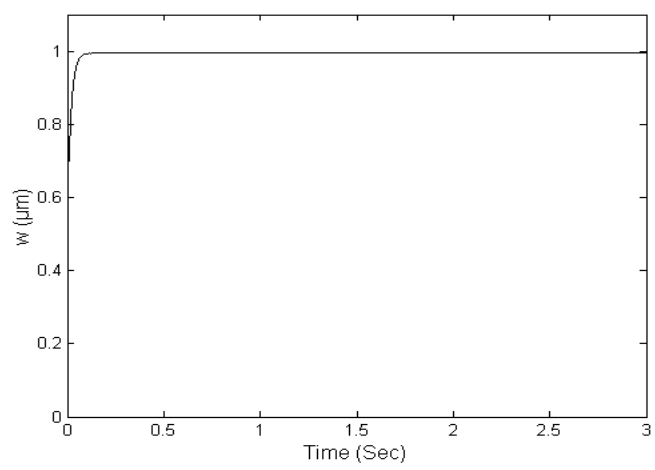

Fig. 10. Step response of the wave amplitude.

\subsection{Torque Control}

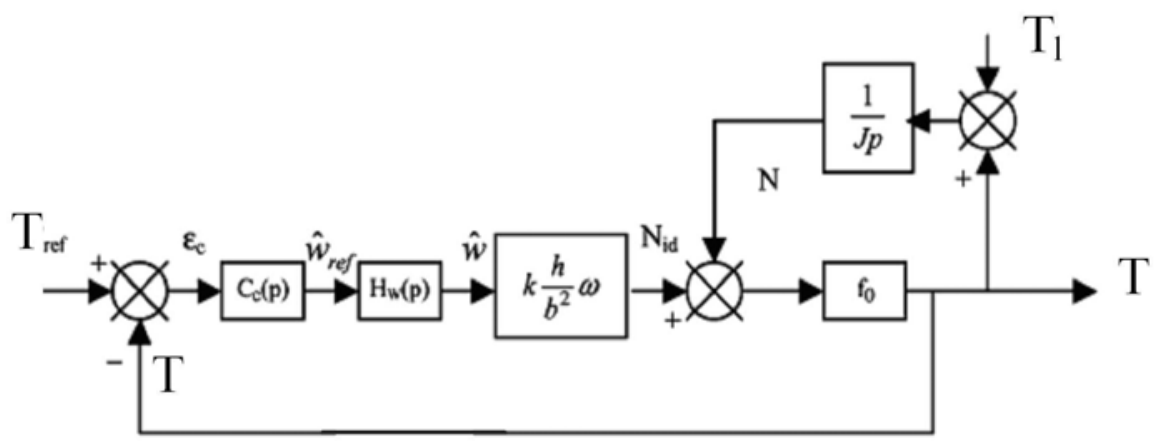

Fig. 11. Outer torque control loop.

The second block of the control scheme of the Figure (11) regulates the torque. The transfer functions in the open loop of the torque is given by (2):

$$
T=H_{w}(s) k \frac{h}{b^{2}} w f_{0} \frac{\frac{J . s}{f_{0}}}{1+\frac{J . s}{f_{0}}} \widehat{W_{r e f}}
$$

The synthesis of the torque controller $T_{l}(s)$ is obtained according to the implementation in the Figure (7).

The transfer function is adjusted by the shaping functions $W_{s 1}, W_{s 2}$. The Figure (12) shows the frequency responses of the compensated TWUM system $W_{s 1} \cdot P_{s} \cdot W_{s 2}$ and the open-loop system $W_{s 1} \cdot P_{s} \cdot W_{s 2} \cdot C_{c}(s)$. Taking into account the low frequency behavior of the motor, with the same method we chose the shaping functions $W_{s 1}, W_{s 2}$ as follows:

$$
W_{s 1}=\frac{585000 s+5}{s} \quad W_{s 2}=1
$$


By using the command ncfsyn of MATLAB $\mu$-Analysis and Synthesis Toolbox, the controller $C_{c}(s)$ ensures correct margins of stability. In Figure (13) the step response of the torque is presented.

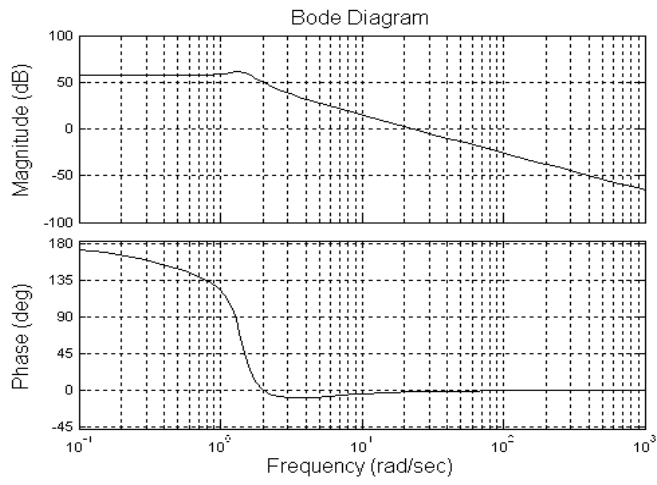

(a) Open-loop responses of $W_{s 1} P_{s} W_{s 2}$

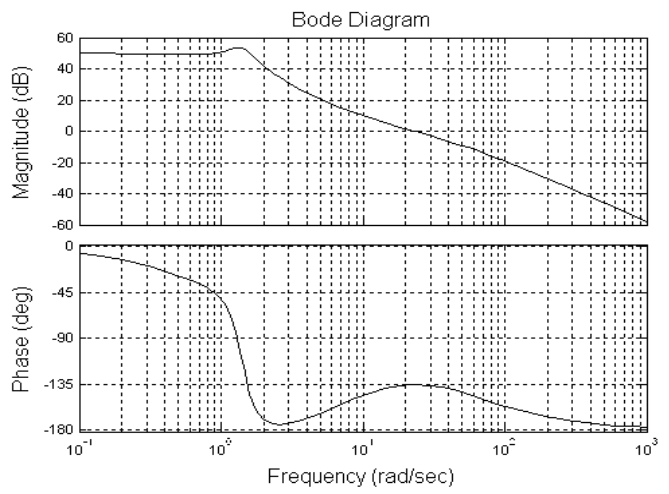

(b) Open-loop responses of $W_{s 1} P_{s} W_{s 2} C_{c}$

Fig. 12. Open-loop responses. 


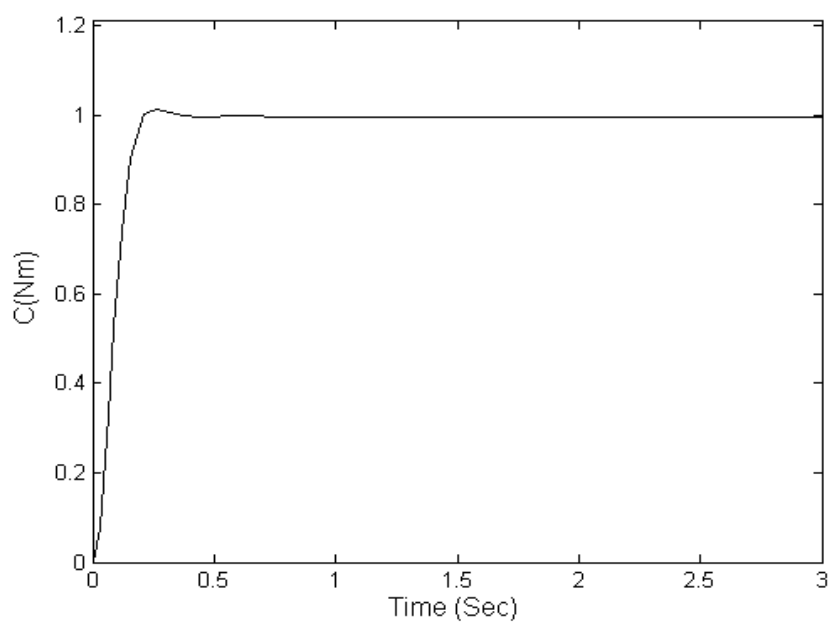

Fig. 13. Step response of motor torque.

\section{Experiments}

Some experiments have been made in order to demonstrate the performances and robustness of the proposed controller. The experimental characterization setup is shown in Fig.14.The rotation is acquired with optoelectric tachometer. An electromagnetic DC brake is used in order to vary the applied torque to the motor shaft. Changing the amplitude of the input voltage and its driving frequency, we can regulate the rotation speed and the stall torque. The normal vibrational amplitude sustained on the stator is measured through a piezoelectric sensing system. The temperature of the stator's surface is acquired in real time via a in - situ temperature sensor (thermocouple). All measurements are acquired through a DSPACE 1100 board connected to ControlDesk graphical user interface software. The $H_{\infty}$ controller has been synthesized and implemented through MATLAB-Simulink 5.5.

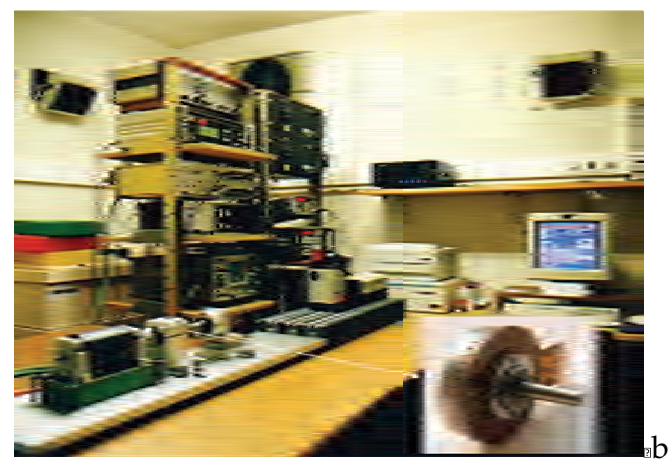

Fig. 14. Experimental setup for USM characterization within harsh environments. 
Nominal and extreme loading conditions have been tested. Motor characteristics of speedtorque shows that the motors rotational speed will decrease with an increase of load. The pressing force $F_{N}$ between the rotor and stator will change both the speed and torque output of the motor notably. Under nominal operating conditions, experiments shows that both the free-load speed and the maximum torque output of the motor will increase nonlinearly with the driving voltage. Without external perturbations and under nominal operating conditions, the proposed cascaded $H_{\infty}$ loop shaping controller demonstrate good position and torque tracking characteristics. The objective of the experiments performed here is to validate the robustness of the proposed controller under various severe disturbances similar to harsh environments, i.e., various inertias, temperature variation and loading torques. As simulated in section V.A, the inner-control loop constituted by the inner $H_{\infty}$ wave amplitude controller demonstrates good robustness when subjected to temperature variations (see Fig.15). Usually, classical PI controller does not offers a good robustness since large temperature variations are at the origin of important stator's resonance frequency shift leading to a decrease of the wave stator amplitude $\hat{W}$. The results shown in Fig.16 demonstrates the good robustness of the proposed controller. Fig.16 shows experiments when considering loaded conditions with inertia added to the rotor to take it up to $5 \mathrm{~kg} . \mathrm{cm}^{2}$ in order to demonstrate the robustness to parameter variation and to investigate its rejection capability while the system encountered large parameter variation (10 times). As shown from experimental results, as long as the torque requirement is satisfied and being kept within the admissible range, the transient response varied negligibly. The steady-state error can be reduced to within 14 mrad.

It is clearly shown that a tradeoff between stability robustness and performance requirements are ensured.

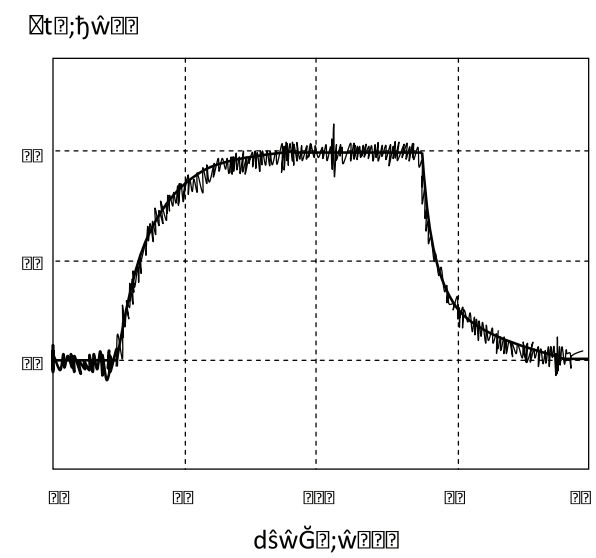

Fig. 15. Step response of stator's wave amplitude $\hat{W}$ when the temperature is settled to $\theta=60$ $\operatorname{deg} C$. 


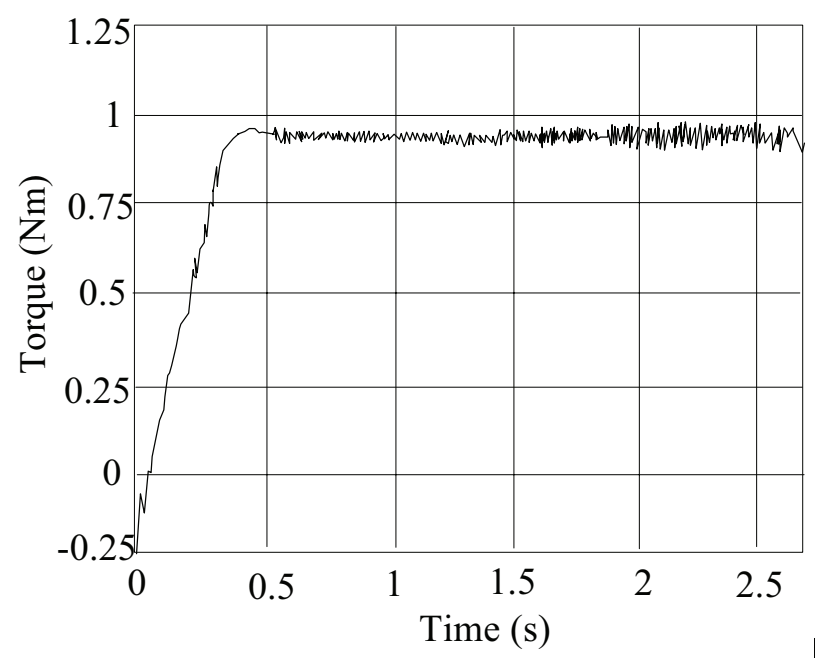

Fig. 16. Step response of driving torque for a load (rotor+load inertia) of $5 \mathrm{~kg} . \mathrm{cm}^{2}$.

\section{Conclusion}

As described in this paper, we showed that the travelling ultrasonic motor technology (TWUM) suits very well mechatronics applications due to its powerful performances to compare with its electrical servomotor counterparts. However, a detailed analysis showed that several operating parameters can greatly influence the operation of TWUM in harsh environments. Based on a causal modeling of TWUM, this paper has successfully implemented a robust control ensuring a good compromise between achievable motor performances (wave amplitude and torque control) and robustness against severe variations of the mechatronic environment. In particular, torque variation has been considered and a loop shaping controller has been determined. Some preliminary experiments have validated the proposed cascaded controllers in real operating conditions. Further experimentation of on-board piezoelectric flight control actuators for hard-launch munitions working on extreme environments are under test in cooperation with Nexter-Bourges company.

\section{Acknowledgment}

The authors gratefully acknowledge the financial support of Nexter Munitions. Moreover, they would like to thank Nexter-Bourges as well as ENSI Bourges staffs for their kindness and support.

\section{References}

[1] T. Morita al " Rotational feedthrough using an ultrasonic motor and its performance in ultra high vacuum conditions" - Vacuum 70 (2003) pages 53-57

[2] F. Giraud, B. Semail, J- Th Audren, " Analysis and Phase Control of a Piezoelectric Travelling-Wave Ultrasonic Motor for Haptic Stick Application " IEEE Transactions On Industry Applications, Vol. 40 No 6, December 2004 
[3] L. Petit al." Frequency behaviour and speed control of piezomotors "- Sensor and Actuator 80 (2000) pages $45-52$

[4] S. Sherrit, "Smart material/actuator needs in extreme environments in space " - SPIE Smart Structures Conference, Vol. 5761-48, 2005

[5] S. Sherrit al, " Resonance Analysis of High Temperature Piezoelectric Materials for Actuation and Sensing ", SPIE Smart Structures Conference, Vol. 5387-58, 2004

[6] D.A. Henderson " Simple Ceramic Motor... Inspiring Smaller Products "International Conference on New Actuators, Actuator 2006

[7] J. Maas T. Schulte and N. Fröhleke, " Model-Based Control for Ultrasonic Motors ", IEEE/ASME Transaction on Mechatronics, Vol 5. No 2, June 2000.

[8] J. Maas, P. Ide, N. Fröhleke, H. Grotstollen, " Simulation Model for Ultrasonic Motors powered by Resonant Converters "Industry Application Conference 1995, IAS'95, Vol. 1, pages 111-120.

[9] J. Maas, T. Schulte, " High Performance Speed Control for Ultrasonic Motors " IEEE/ASME International Conference on Advanced Intelligent Mechatronics, 1999

[10] S. Font, G. Duc, F. Carrière, " Commande fréquentielle robuste, Application aux paliers magnétiques" Techniques de l'ingnieur, R 7432.

[11] J.S Chen, I.D Lin, " Toward the implementation of an ultrasonic motor servo drive using FPGA " Mechatronics 12 (2002), pages 511-524

[12] G. Bal, " A Digitally Controlled Drive System for Travelling-wave Ultrasonic Motor " Turk J Elec Engin, Vol.11, pages 155 - 168

[13] D. McFarlane, K. Glover, " An $H_{\infty}$ design procedure using robust stabilization of normalized coprime factors ", IEEE Conference on Decision and Control, Austin,TX,1988.

[14] D. McFarlane, K. Glover, " Robust controller design using normalised coprime factor plant descriptions ", lecture Notes in Control and information Sciences, vol. 138, New York: Springer Verlag, 1989

[15] G. J. Balas, J. C. Doyle, K. Glover, A. Packard, and R. Smith, $\mu$-Analysis and Synthesis Toolbox. Natick, MA: The MathWorks, Inc, 1994.

[16] P. Lundstrom, S. Skogstad, Z.C. Wang, "Weight selection for H-infinity and mu-control methods: insights and examples from process control," Workshop on $\mathrm{H}_{\infty}$ control, Brighton, U.K., pp.139-1991.

[17] Y. Bar-Cohen, X. Bao,W. Grandia, "Rotary Ultrasonic Motors Actuated By Travelling Flexural Waves", SPIE 6th Annual International Symposium on Smart Structures and Materials, March 1999

[18] R.J. Wai, K.H. Su, " Supervisory Control for Linear Piezoelectric Ceramic Motor Drive Using Genetic Algorithm "IEEE Transactions on Industrial Electronics, Vol.53, No2, April 2006 


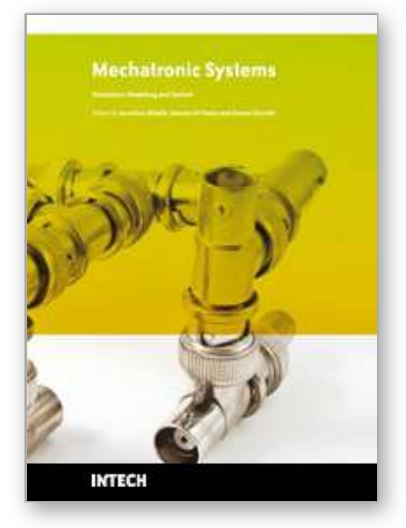

\author{
Mechatronic Systems Simulation Modeling and Control \\ Edited by Annalisa Milella Donato Di Paola and Grazia Cicirelli
}

ISBN 978-953-307-041-4

Hard cover, 298 pages

Publisher InTech

Published online 01, March, 2010

Published in print edition March, 2010

This book collects fifteen relevant papers in the field of mechatronic systems. Mechatronics, the synergistic blend of mechanics, electronics, and computer science, integrates the best design practices with the most advanced technologies to realize high-quality products, guaranteeing at the same time a substantial reduction in development time and cost. Topics covered in this book include simulation, modelling and control of electromechanical machines, machine components, and mechatronic vehicles. New software tools, integrated development environments, and systematic design methods are also introduced. The editors are extremely grateful to all the authors for their valuable contributions. The book begins with eight chapters related to modelling and control of electromechanical machines and machine components. Chapter 9 presents a nonlinear model for the control of a three-DOF helicopter. A helicopter model and a control method of the model are also presented and validated experimentally in Chapter 10. Chapter 11 introduces a planar laboratory testbed for the simulation of autonomous proximity manoeuvres of a uniquely control actuator configured spacecraft. Integrated methods of simulation and Real-Time control aiming at improving the efficiency of an iterative design process of control systems are presented in Chapter 12. Reliability analysis methods for an embedded Open Source Software (OSS) are discussed in Chapter 13. A new specification technique for the conceptual design of self-optimizing mechatronic systems is presented in Chapter 14 . Chapter 15 provides a general overview of design specificities including mechanical and control considerations for micro-mechatronic structures. It also presents an example of a new optimal synthesis method to design topology and associated robust control methodologies for monolithic compliant microstructures.

\title{
How to reference
}

In order to correctly reference this scholarly work, feel free to copy and paste the following:

Moussa Boukhnifer, Antoine Ferreira and Didier Aubry (2010). Robust Control of Ultrasonic Motor Operating under Severe Operating Conditions, Mechatronic Systems Simulation Modeling and Control, Annalisa Milella Donato Di Paola and Grazia Cicirelli (Ed.), ISBN: 978-953-307-041-4, InTech, Available from: http://www.intechopen.com/books/mechatronic-systems-simulation-modeling-and-control/robust-control-ofultrasonic-motor-operating-under-severe-operating-conditions

\section{INTECH}

open science | open minds

\author{
InTech Europe \\ University Campus STeP Ri
}

\section{InTech China}

Unit 405, Office Block, Hotel Equatorial Shanghai 
Slavka Krautzeka 83/A

51000 Rijeka, Croatia

Phone: +385 (51) 770447

Fax: +385 (51) 686166

www.intechopen.com
No.65, Yan An Road (West), Shanghai, 200040, China 中国上海市延安西路65号上海国际贵都大饭店办公楼405单元 Phone: +86-21-62489820

Fax: $+86-21-62489821$ 
(C) 2010 The Author(s). Licensee IntechOpen. This chapter is distributed under the terms of the Creative Commons Attribution-NonCommercialShareAlike-3.0 License, which permits use, distribution and reproduction for non-commercial purposes, provided the original is properly cited and derivative works building on this content are distributed under the same license. 